\title{
Erratum
}

\section{Efficient Protocol for the Phosphine-Free Suzuki-Miyaura Reaction Catalyzed by Palladium on Carbon at Room Temperature}

Guolin Zhang* Synthesis 2005, 537.

The author list and contact information for this manuscript were incomplete. The results reported in this Letter were all carried out in the Laboratory of Medicinal Chemistry, Gifu Pharmaceutical University, Japan under the supervision of Professors Hironao Sajiki and Kosaku Hirota. The single author, Guolin Zhang, was a visiting research associate during one year at that laboratory. The results were illegally published by Guolin Zhang without authorization by Professors Hironao Sajiki and Kosaku Hirota and Gifu Pharmaceutical University. The author apologizes for misrepresenting the research covered in this paper as his work alone.

Therefore, the correct information is as follows:

\section{Efficient Protocol for the Phosphine-Free Suzuki-Miyaura Reaction Catalyzed by Palladium on Carbon at Room Temperature}

Hironao Sajiki,* Takanori Kurita, Atsushi Kozaki, Guolin Zhang, ${ }^{\dagger}$ Yoshiaki Kitamura, Tomohiro Maegawa, Kosaku Hirota*

Laboratory of Medicinal Chemistry, Gifu Pharmaceutical University, Mitahora-higashi, Gifu 502-8585, Japan

Fax +81(58)2375979; E-mail: sajiki@gifu-pu.ac.jp

${ }^{\dagger}$ A visiting research associate on leave, current address: Department of Medicinal Chemistry, College of Pharmaceutical Sciences, Zhejiang University, Hangzhou 310031, P. R. China 